\title{
Strongly nonlinear steepening of long interfacial waves
}

\author{
N. Zahibo ${ }^{1}$, A. Slunyaev ${ }^{2}$, T. Talipova ${ }^{2}$, E. Pelinovsky ${ }^{2,3}$, A. Kurkin ${ }^{3}$, and O. Polukhina ${ }^{2,3}$ \\ ${ }^{1}$ Département de Physique, Université des Antilles et de la Guyane, Guadeloupe, France \\ ${ }^{2}$ Department of Nonlinear Geophysical Processes, Institute of Applied Physics, Nizhny Novgorod, Russia \\ ${ }^{3}$ Department of Applied Mathematics, Nizhny Novgorod Technical State University, Nizhny Novgorod, Russia
}

Received: 23 February 2007 - Revised: 10 May 2007 - Accepted: 16 May 2007 - Published: 8 June 2007

\begin{abstract}
The transformation of nonlinear long internal waves in a two-layer fluid is studied in the Boussinesq and rigid-lid approximation. Explicit analytic formulation of the evolution equation in terms of the Riemann invariants allows us to obtain analytical results characterizing strongly nonlinear wave steepening, including the spectral evolution. Effects manifesting the action of high nonlinear corrections of the model are highlighted. It is shown, in particular, that the breaking points on the wave profile may shift from the zerocrossing level. The wave steepening happens in a different way if the density jump is placed near the middle of the water bulk: then the wave deformation is almost symmetrical and two phases appear where the wave breaks.
\end{abstract}

\section{Introduction}

Internal waves in a stratified fluid are an important object for study in geophysical flows, for example in lakes, rivers, oceans and the atmosphere (Baines, 1995; Grimshaw, 2002; Miropolsky, 2002; Heilfrich and Melville, 2006). Breaking internal waves contribute to the mixing of salt, heat and tracers in water (Henyey and Hoering, 1997; Munk and Wunsh, 1998; Chant and Wilson, 2000; Horn et al., 2001; Fringer and Street, 2003). There are many observations and theoretical models of bore-like shapes of long internal waves on ocean shelves, and in fjords and lakes (Klemp et al., 1997; Afanasyev and Pelter, 2000; Armi and Farmer, 2001; Holland et al., 2002). In many cases these strongly nonlinear internal waves are described in the framework of two-layer flows; see, for instance, the books by Baines (1995) and Lyapidevsky and Teshukov (2000).

Indeed, two classical scenarios for the eventual mixing by interfacial waves in this model are possible: they are

Correspondence to: A. Slunyaev

(slunyaev@hydro.appl.sci-nnov.ru) shear-instability and overturning of internal waves. Shearinstability occurs in the linear and nonlinear limits (Craik, 1985; Baines, 1995). However, Milewski et al. (2004) showed that long nonlinear interfacial waves are stable as long as the flow remains smooth. Therefore, wave breaking turns out to be the main source of mixing, so its understanding becomes necessary. These processes have not been studied for large-amplitude waves. Here we consider the deformation of strongly nonlinear periodic (sinusoidal) interfacial waves in a two-fluid system bounded by rigid walls in the case of a small density jump (the Boussinesq approximation).

The paper is organized as follows. The Riemann invariants describing the interfacial wave dynamics are discussed in Sect. 2. The unidirectional description for a rightward propagating wave is developed in Sect. 3; the fully nonlinear expression for the wave speed is given, and the ranges of variability of the fluid and waves velocities are obtained with the help of a trigonometric representation. Section 4 describes the strongly nonlinear wave evolution resulting in steepening and the formation of breaking points on the wave profile. The corresponding spectral evolution is analyzed in Sect. 5, both numerically and with help of weakly nonlinear approximate solutions. The conclusion collects the main results of this study.

\section{Riemann invariants for a two-layer fluid}

In this section the problem is formulated and the derivation of the governing equations is given. Here we follow mainly Baines (1995) and Lyapidevsky and Teshukov (2000), combining the physical evidence and mathematical elegance of these references.

Let us consider a two-layer system bounded by rigid walls (see Fig. 1). The fluid in the layers is supposed to be homogeneous, inviscid and incompressible. In the long-wave

Published by Copernicus Publications on behalf of the European Geosciences Union and the American Geophysical Union. 


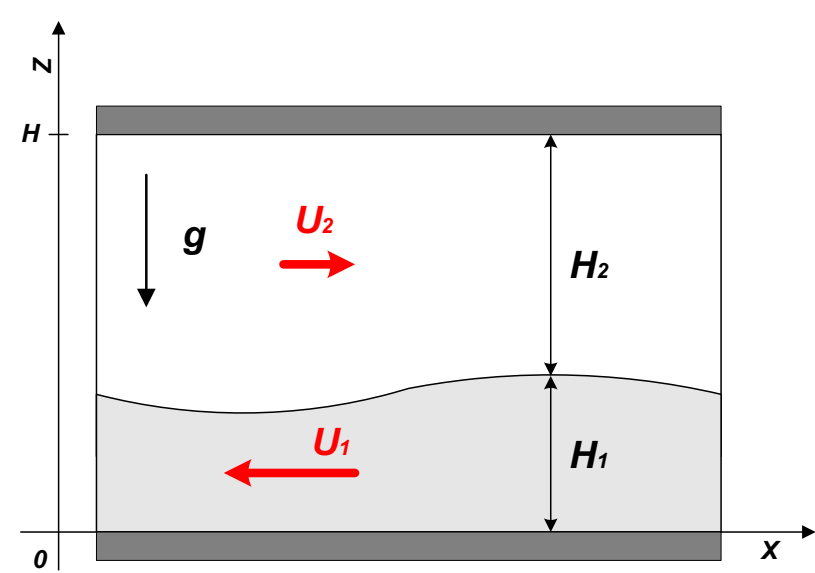

Fig. 1. Configuration of the problem.

approximation the hydraulic approach may be applied, and then the governing system of equations is

$\rho_{j}\left(\frac{\partial U_{j}}{\partial T}+U_{j} \frac{\partial U_{j}}{\partial X}\right)+\frac{\partial p_{j}}{\partial X}=0$,

$\frac{\partial H_{j}}{\partial T}+\frac{\partial}{\partial X}\left(H_{j} U_{j}\right)=0$.

Subscript $j=1,2$ numerates the layers; $U_{j}$ are the horizontal fluid velocities in the layers each of constant density $\rho_{j}$, $p_{j}$ are the hydrostatic pressures, $H_{j}(X, T)$ are the variable layer thicknesses, while the total water depth remains constant: $H_{1}+H_{2}=H$.

Adding the two mass conservation Eq. (2) gives the conservation of the total volume flux. Supposing that the undisturbed water does not move then

$U_{1} H_{1}+U_{2} H_{2}=0$.

Thus the flows in this problem may be described by a single variable, $W(X, T)=\left(U_{2}-U_{1}\right) / 2$, and the interface displacement is characterized by the difference variable $\Delta H=H_{1}-H_{2}$ so that

$U_{1}=-2 W \frac{H_{2}}{H}, \quad U_{2}=2 W \frac{H_{1}}{H} \quad$ and

$H_{1}=\frac{H+\Delta H}{2}, \quad H_{2}=\frac{H-\Delta H}{2}$.

Subtraction of the conservation Eq. (2) then gives

$\frac{\partial}{\partial T} \Delta H+\frac{\partial}{\partial X}\left(W \frac{\Delta H^{2}-H^{2}}{H}\right)=0$.

The other equation results from subtracting Eq. (1)

$$
\begin{aligned}
& \frac{\partial}{\partial T}\left(U_{1}-\frac{\rho_{2}}{\rho_{1}} U_{2}\right)+\frac{1}{2} \frac{\partial}{\partial X} \\
& \quad\left(\left(U_{1}^{2}-\frac{\rho_{2}}{\rho_{1}} U_{2}^{2}\right)-\frac{\rho_{1}-\rho_{2}}{\rho_{1}} g\left(H_{2}-H_{1}\right)\right)=0,
\end{aligned}
$$

where $g$ is the gravity acceleration that comes from the hydrostatic pressure solution. In the Boussinesq approximation $\rho_{1} \approx \rho_{2} \approx \rho$, Eq. (6) tends to the second equation for the variables $W$ and $\Delta H$ that with Eq. (5) completes the system:

$$
\begin{aligned}
& \frac{\partial}{\partial T} W+\frac{\partial}{\partial X}\left[\left(W^{2}-C^{2}\right) \frac{\Delta H}{H}\right]=0, \\
& C=\frac{1}{2} \sqrt{g \frac{\Delta \rho}{\rho} H}, \quad \Delta \rho=\rho_{1}-\rho_{2} .
\end{aligned}
$$

The constant $C$ in (7) is equal to the maximum speed of a linear interfacial wave in the two-layer system

$C_{\text {lin }}=\sqrt{g \frac{\Delta \rho}{\rho} \frac{H_{1} H_{2}}{H}}$

when the layers have equal depths $H_{1}=H_{2}$. The values $C$ and $H$ naturally define the typical scales for the problem (5) and (7). After normalizing

$W \rightarrow C w, \quad \Delta H \rightarrow H \delta, \quad T \rightarrow \frac{H}{C} t, \quad X \rightarrow H x$,

the system (5) and (7) becomes (Lyapidevsky and Teshukov, 2000)

$$
\begin{aligned}
& \frac{\partial \delta}{\partial t}+\frac{\partial}{\partial x}\left(w\left(\delta^{2}-1\right)\right)=0, \\
& \frac{\partial w}{\partial t}+\frac{\partial}{\partial x}\left(\delta\left(w^{2}-1\right)\right)=0 .
\end{aligned}
$$

It is remarkable that this system may always be reduced to the surface wave equations. Indeed, after the substitution

$\gamma=2 w \delta, \quad \lambda=\left(1-w^{2}\right)\left(1-\delta^{2}\right)$,

the system (10) may be straightforwardly transformed to

$\frac{\partial \gamma}{\partial t}+\gamma \frac{\partial \gamma}{\partial x}+\frac{\partial \lambda}{\partial x}=0, \quad \frac{\partial \lambda}{\partial t}+\frac{\partial}{\partial x}(\gamma \lambda)=0$,

where $\gamma$ and $\lambda$ correspond to the shallow surface waves fluid velocity and the water elevation respectively.

The system (12) is known to be hyperbolic and it may be represented in terms of the Riemann invariants

$V_{ \pm}=\gamma \pm \sqrt{\lambda}, \quad S_{ \pm}=\gamma \pm 2 \sqrt{\lambda}$,

that allows us to write the governing system of equations as

$\frac{\partial S_{ \pm}}{\partial t}+V_{ \pm} \frac{\partial S_{ \pm}}{\partial x}=0$.

Using (11), the expressions (13) define the Riemann invariants for the case of an interfacial wave, as

$S_{ \pm}=2 w \delta \pm 2 \sqrt{\left(1-\delta^{2}\right)\left(1-w^{2}\right)}$,

$V_{ \pm}=2 w \delta \pm \sqrt{\left(1-\delta^{2}\right)\left(1-w^{2}\right)}$. 
The formulae (15) and (16) in different forms were presented in Baines (1995), Lyapidevsky and Teshukov (2000) and Slunyaev et al. (2003), and form the basis of our research.

The characteristics of this hyperbolic system (the velocities $V_{ \pm}$) may be expressed as smooth functions of the Riemann invariants. This fact was used by Milewski et al. (2004) to prove the nonlinear stability of smooth disturbances: the system remains hyperbolic while the waves are smooth. They report that unforced flows cannot reach the threshold of shear-instability, at least without breaking first. The fully nonlinear steepening of a wave will be considered in Sect. 4 . To do this the expression for the nonlinear wave velocity is obtained in the next section.

\section{Unidirectional fully nonlinear interfacial waves}

With the help of the Riemann invariants representation (14)(16) the fully nonlinear model for unidirectional internal waves in a two-layer system may be easily found. The form of expressions (15) and (16) suggests using the trigonometric substitution; let us introduce new variables as

$\delta=\sin (\varphi), \quad|\varphi| \leq \frac{\pi}{2}, \quad w=\sin (\psi), \quad|\psi| \leq \frac{\pi}{2}$.

Then the representation of the Riemann invariants may be formulated as

$S_{ \pm}= \pm 2 \cos (\varphi \mp \psi)$

$V_{ \pm}=S_{ \pm}-\cos (\varphi) \cos (\psi)$.

The Riemann invariants $S_{ \pm}$are preserved along corresponding characteristics moving with velocities $V_{ \pm}$and thereby build the wave field. Let us consider a single perturbation propagating to the right, thus, suppose the leftward propagating invariant $S_{-}$to be constant. If the case when there is no shear flow $(w=0)$ is considered, then the condition of the invariant conservation gives

$\varphi+\psi=\varphi_{0}, \quad \delta_{0}=\sin \left(\varphi_{0}\right)$.

Here $\delta_{0}$ is the unperturbed dimensionless difference of the layers depths. After using relation (18) the advection may be straightforwardly obtained as

$w=\sin \left(\varphi_{0}-\varphi\right)=2\left(h_{10}-h_{20}\right) \sqrt{h_{1} h_{2}}-2\left(h_{1}-h_{2}\right) \sqrt{h_{10} h_{20}}$.

The values $h_{10}$ and $h_{20}$ in (19) are the unperturbed dimensionless depths of the layers:

$h_{10,20}=\frac{1 \pm \sin \varphi_{0}}{2}$,

$h_{1}$ and $h_{2}$ are the dimensionless current depths, such as $h_{1}=h_{10}+\eta$ and $h_{2}=h_{20}-\eta$, where $\eta(x, t)$ is the dimensionless interface displacement.
The reality of the solution naturally provides the condition $|w| \leq 1(|W| \leq C)$. It follows from (19), that function $w(\eta)$ is not monotonic. It is clearly seen from (17) and (19) that it defines an arc of a circle in the plane $(\delta, w)$; the value $\varphi_{0}$ plays the role of a phase shift between the oscillations of the interface $\eta$ and the advection $w$. The function $w$ reaches its extremes when

$\varphi=\varphi_{0} \pm \pi / 2$ or otherwise

$\eta= \pm \sqrt{h_{10} h_{20}}-\frac{h_{10}-h_{20}}{2}$.

The signs + or - in (21) should be defined according to the sign of the difference $h_{10}-h_{20}$.

The velocities of the flows $U_{1}$ and $U_{2}$ may be straightforwardly obtained from (4) and (19) as (in the dimensionless form)

$u_{2,1}=\sin \left(\varphi_{0}-\varphi\right)(\sin \varphi \pm 1)$.

It is certainly seen from (22), that the physical fluid velocity may exceed the value $C$. The extremes of (22) may be found and then the following restriction takes place: $\left|u_{2,1}\right| \leq 2$. These velocities are plotted in Fig. 2 by the blue dashed $\left(u_{2}\right)$ and the red dash-dotted $\left(u_{1}\right)$ lines. The vertical axis in Fig. 2 corresponds to the position of the interface $h_{1}$, whereas the horizontal solid line shows the undisturbed level $h_{10}$. The flow velocities have different signs due to the conservation law (3). The flow in the layer is opposite to the linear wave propagation if the displacement makes this layer shallower.

Since the advection and interface displacement are constrained according to (19), then the Riemann invariants (15) may be expressed as functions of one variable, and therefore (14) gives the evolution equation for the rightward propagating wave

$\frac{\partial \eta}{\partial t}+V_{+} \frac{\partial \eta}{\partial x}=0$

where the explicit dimensionless form of the full nonlinear velocity follows from $\left(16^{*}\right)$ and may be written in the form:

$$
\begin{aligned}
& V_{+}=\frac{3}{2} \cos \left(2 \varphi-\varphi_{0}\right)-\frac{1}{2} \cos \left(\varphi_{0}\right)=2 \sqrt{h_{10} h_{20}} \times \\
& {\left[1-3\left(h_{1}-h_{2}\right)^{2}\right]+6\left(h_{10}-h_{20}\right)\left(h_{1}-h_{2}\right) \sqrt{h_{1} h_{2}} .}
\end{aligned}
$$

This formula apparently was first presented in (Slunyaev et al., 2003). A similar approach was used by Ostrovsky and Grue (2003) resulting in an implicit model. The curves (24) are plotted in Fig. 2 by thick black solid lines. The value $V_{+}$remains bounded: $\left|V_{+}\right| \leq 2$; it reaches the minimum value $\left(V_{+}=-2\right)$ if

$h_{10}=\frac{1}{2} \quad$ and $\quad \eta= \pm \frac{1}{2}$.

It corresponds to the situation when in an equal-depth layered fluid the interface touches the bottom or the top (Fig. 2c). The positive maximum value is limited by

$V_{+} \leq \frac{3}{2}-\sqrt{h_{10} h_{20}}$; 


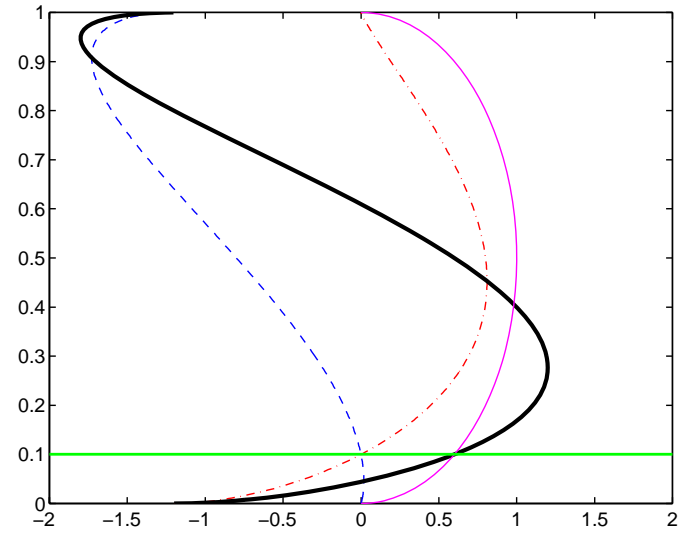

(a)

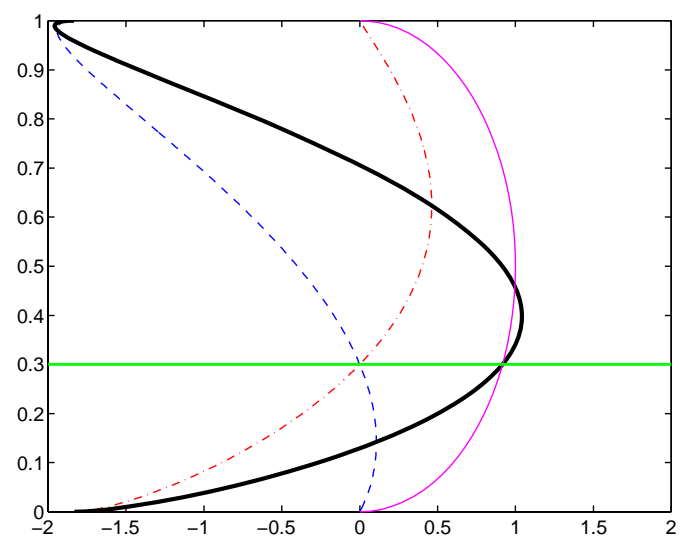

(b)

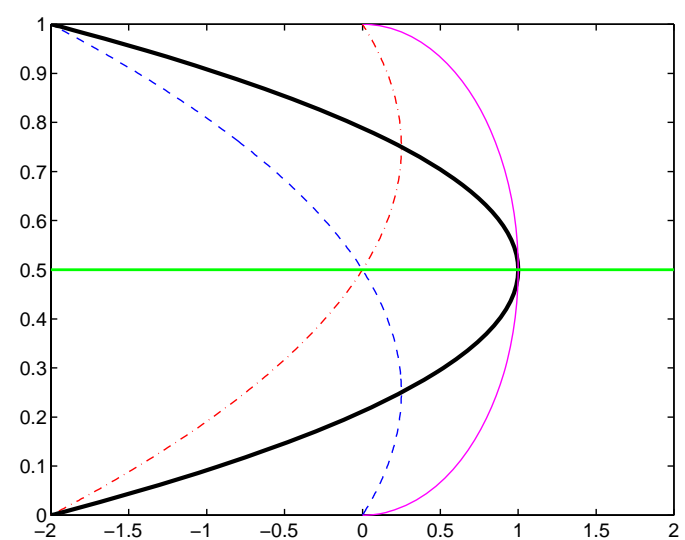

(c)

Fig. 2. Velocities in the two-layer fluid. The vertical axis measures the position of the interface $h_{1}$; the horizontal line shows the rest level $h_{10}: 0.1$ (a), 0.3 (b) and 0.5 (c). The horizontal axis measures the dimensionless velocities $u_{1}$ (red dash-dot), $u_{2}$ (blue dash), linear wave speed $V_{\text {lin }}\left(h_{1}\right)$ (thin pink solid line) and the full nonlinear velocity $V_{+}$(thick black solid line). thus it is always less than $3 / 2$, and reaches this value when one of the layers vanishes. It is seen from Fig. 2 that the wave velocity may then reach the fluid speeds in the layers.

The linear velocity (limit $\eta \rightarrow 0$ ) is very well known:

$V_{\text {lin }}=2 \sqrt{h_{10} h_{20}}$,

which is the dimensionless form of (8). This velocity is shown by thin pink solid lines in Fig. 2 as function of the local interface displacement (i.e., $V_{\text {lin }}=2\left(h_{1} h_{2}\right)^{\frac{1}{2}}$ in the figures). It follows from (24), that $V_{+}(\eta)=V_{\operatorname{lin}}(\eta=0)$ in two cases: when $\eta=0$ and when $\eta=\left(h_{20}-h_{10}\right) / 2$ (in other words, in the middle of the fluid bulk).

The nonlinear velocity (24) passes zero when the interface displacements are

$\eta_{1}=-\frac{1}{\sqrt{3}} h_{10}$ and $\eta_{2}=\frac{1}{\sqrt{3}}-\frac{1}{\sqrt{3}} h_{10}$,

thus, the nonlinear velocity of a large enough interfacial displacement becomes negative in contrast to the speeds of weak perturbations, which should lead to a significant change of the shape of an intense wave.

Expression (24) may be easily decomposed into the Taylor series

$V_{+}=V_{\text {lin }}\left(1+\alpha \eta+\alpha_{1} \eta^{2}+\alpha_{2} \eta^{3}+\alpha_{3} \eta^{4}+\ldots\right)$.

with coefficients

$\alpha=-\frac{3}{2} \frac{h_{10}-h_{20}}{h_{10} h_{20}}, \quad \alpha_{1}=-\frac{3}{8} \cdot \frac{1+4 h_{10} h_{20}}{\left(h_{10} h_{20}\right)^{2}}$,

$\alpha_{2}=-\frac{3}{16} \frac{h_{10}-h_{20}}{\left(h_{10} h_{20}\right)^{3}}, \quad \alpha_{3}=-\frac{15}{128} \frac{\left(h_{10}-h_{20}\right)^{2}}{\left(h_{10} h_{20}\right)^{4}}$.

The expansions of the nonlinear wave velocity (29) build asymptotic nonlinear evolution equations for internal waves. The two first coefficients $\alpha_{1}$ and $\alpha_{2}$ are well-known: they define the nonlinear term in the classical Korteweg - de Vries equation and its generalization (so-called Gardner equation) (Kakutani and Yamasaki, 1978). The next two coefficients in (29) were found for the generalization of the Gardner equation via asymptotic expansions (Polukhina and Slunyaev, 2006) and coincide with the ones given by (30). Different approximations of the full nonlinear models were also studied recently by Sakai and Redekopp (2007).

It is interesting to note that all terms of the Taylor series (29) tend to zero when $h_{10}=h_{20}$ except the cubic nonlinearity, and the nonlinear velocity then becomes purely polynomial (parabolic):

$V_{+}=1-12 \eta^{2}$.

When the thickness of the bottom layer is less than the thickness of the upper layer $\left(h_{10}<h_{20}\right)$, the nonlinear coefficients of both even orders in (29) are positive and change their signs when the interface lies in the middle of water bulk. Both coefficients of odd orders are negative for the two-layer stratification. The case $h_{10}<h_{20}$ will be analyzed in the next section when studying the wave deformation. The other case may be easily obtained due to the symmetry of the problem. 

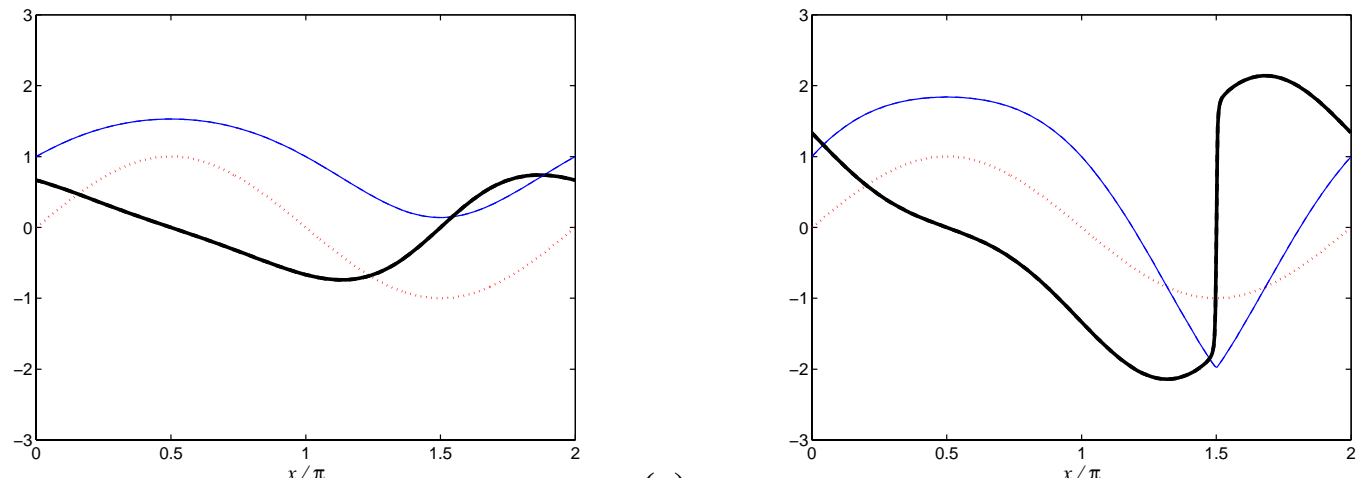

(a)

(b)
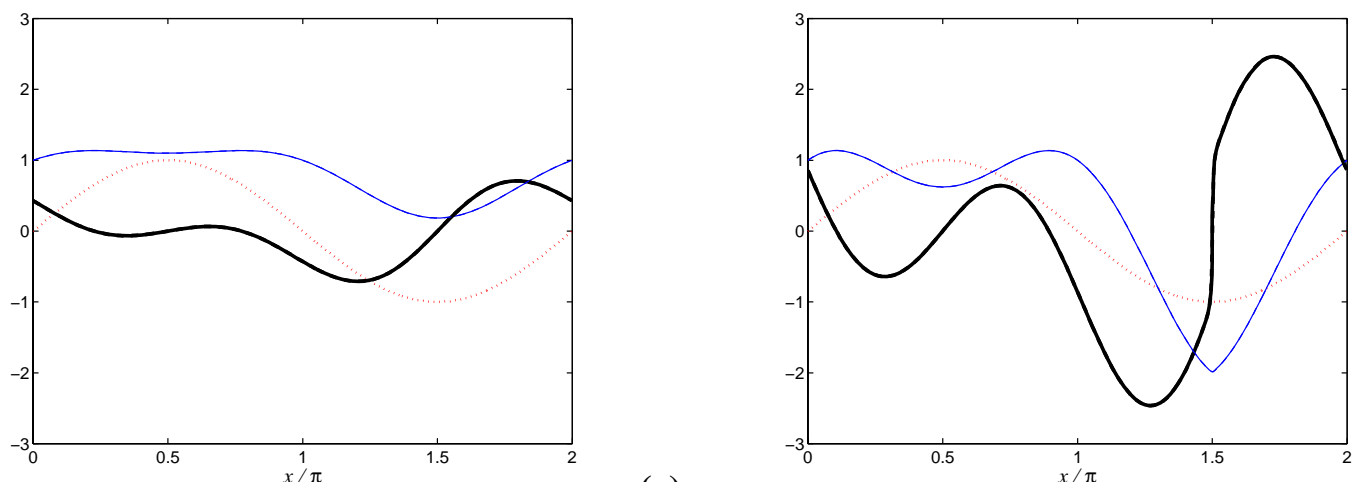

(c)

(d)
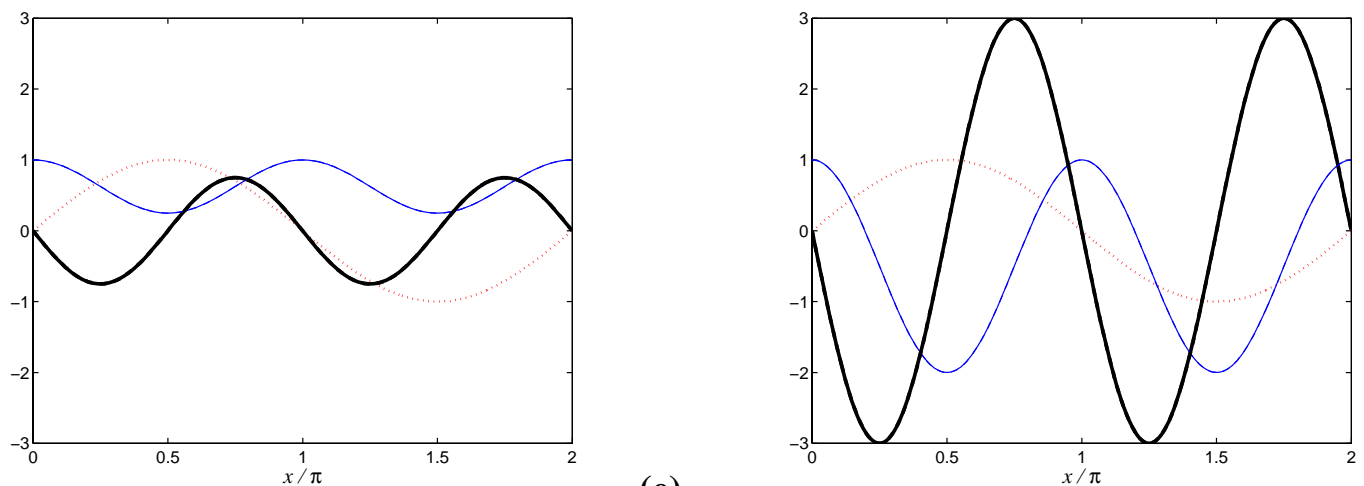

(e)

Fig. 3. Profiles of the normalized wave displacement $\eta_{0} / a$ (dotted red line), nonlinear wave velocity $V_{0} / V_{\text {lin }}$ (thin blue curves) and its derivative $d V_{0} / d x / V_{\text {lin }}$ (thick black curves) for different depths of the layers: $h_{10}: 0.1$ (a, b), 0.3 (c, d) and 0.5 (e, f) and intensity of the waves: $a=h_{10} / 2(\mathrm{a}, \mathrm{c}, \mathrm{e})$ and $a=h_{10}(\mathrm{~b}, \mathrm{~d}, \mathrm{f})$.

\section{Nonlinear deformation of an internal wave}

The process of nonlinear deformation of a simple wave leading to its breaking is classical (see for instance Whitham (1974)), but is typically considered in the case of weak (quadratic) nonlinearity. Consideration of the fully nonlinear case does not differ significantly from the weakly nonlinear theory after the exact expression for the nonlinear velocity is written down (24), and is studied hereafter.
Let us consider the initial-value problem, i.e., that the wave is defined at the initial moment of time $t=0$ : $\eta(x, t=0)=\eta_{0}(x)$. Then the initial distribution of the nonlinear velocity is given by $V_{0}(x)=V_{+}\left(\eta_{0}(x)\right)$. The solution is governed by Eq. (23) and so

$\eta(x, t)=\eta_{0}\left(x-V_{+} t\right)$.

The solution (32) represents the well-known simple or Riemann wave (Whitham, 1974). It describes the process of 
nonlinear wave deformation with the steepening of the wave front. The steepness may be found by differentiation of (32)

$$
\frac{\partial \eta}{\partial x}=\frac{\eta_{0}^{\prime}}{1+t V_{0}^{\prime}}
$$

where primes mean the total derivative by $x$. It is clear that the steepness singularity $\frac{\partial \eta}{\partial x} \rightarrow \infty$ arises for the first time when the denominator in (33) becomes zero, which needs $d V_{0} / d x<0$. The denominator in (33) decreases with time and reaches zero for the first time at the moment $t=t_{b r}$,

$t_{b r}=\frac{1}{\max \left(-V_{0}^{\prime}\right)}$.

The wave starts breaking at the point of the maximum derivative of the nonlinear velocity, which is not necessarily the point of maximum initial steepness.

Let us consider the breaking of a sinusoidal internal wave $\eta_{0}(x)=a \sin (x)$ as an example with the initial maximum steepness $s_{0}=a$ at the coordinate origin $x=0$. The derivative of the nonlinear wave velocity is

$$
\frac{\partial V_{+}}{\partial x}=D \frac{\partial \eta}{\partial x}, \quad D=\frac{\partial V_{+}}{\partial \eta}=-\frac{6 \sin \left(2 \varphi-\varphi_{0}\right)}{\cos (\varphi)} .
$$

The function $D$ in (35) depends on $x$ and $t$ via $h_{1}$ and $h_{2}$ :

$$
\begin{gathered}
D\left(h_{1}, h_{2}\right)=12\left(h_{10}-h_{20}\right) \sqrt{h_{1} h_{2}}-24\left(h_{1}-h_{2}\right) \\
\sqrt{h_{10} h_{20}}-3 \frac{\left(h_{10}-h_{20}\right)\left(h_{1}-h_{2}\right)^{2}}{\sqrt{h_{1} h_{2}}} .
\end{gathered}
$$

Considering the sinusoidal Riemann wave, (35) becomes

$\frac{d V_{0}}{d x}=a \cos (x) \cdot D\left(h_{1}=h_{10}+\eta_{0}, h_{2}=h_{20}-\eta_{0}\right)$.

The weakly-nonlinear case straightforwardly follows from the latter formulae when we put $D=D\left(h_{10}, h_{20}\right)$. Then the expression for the nonlinear velocity is given by (29) when only the quadratic nonlinear term is taken into account. The quantity $D$ does not depend on the coordinate and the first breaking point corresponds to the minimum of the cosine function ( $D>0$ if $h_{1}<h_{2}$, see (30)). That is, in the weakly nonlinear case the breaking occurs at phase $x=\pi$, where the wave shape crosses the undisturbed (zero) level.

The shapes of the steep waves, the nonlinear wave velocity and its derivative are given in Fig. 3 for three different thickness of the bottom layer: $h_{10}=0.1(\mathrm{a}, \mathrm{b}), 0.3(\mathrm{c}, \mathrm{d})$, and $0.5(\mathrm{e}, \mathrm{f})$. The amplitude of the sinusoidal wave is also different: moderate $a=h_{10} / 2$ (a, c, e) and maximum $a=h_{10}$ (b, $\mathrm{d}, \mathrm{f}$ ). It is seen that the wave velocity deviates from the linear value when the wave amplitude grows. It becomes sharp when the amplitude is maximal so that the derivative of the velocity jumps in the wave trough. It may be also observed in Fig. 3 how the position of the minimum of the derivative shifts rightward $x=\pi$. The reverse movement of the trough is very important for intense waves and may be even faster than the forward movement of the wave (see Fig. 2). Acceleration of the wave is achieved mainly in the case when one layer is much thinner than the other (see the blue thin curves in Fig. 3). In the case $h_{10}=0.5$ the wave becomes symmetric (Figs. 3e, f) and both the crest and the trough move backward. Two minima of the derivative of the nonlinear wave velocity appear that should lead to the formation of two breaking points on the wave profile per wave period.

The position of the first breaking point is defined as the minimum of the derivative of the nonlinear velocity $V_{0}$ (see (33)). Letting the second derivative of $V_{0}$ equal to zero, one may obtain the relation between wave amplitude $a$ and the interface displacement at the point of the first breaking $\eta_{b r}$, which in the trigonometrical form reads

$$
\begin{aligned}
& a=\sqrt{\eta_{b r}^{2}+\frac{\eta_{b r} \cos \left(\varphi_{b r}\right)}{2 \tan \left(\varphi_{b r}\right)+4 \cot \left(2 \varphi_{b r}-\varphi_{0}\right)},} \\
& \eta_{b r}=\frac{1}{2}\left(\sin \left(\varphi_{b r}\right)-\sin \left(\varphi_{0}\right)\right)
\end{aligned}
$$

It is evident from Fig. 3 that $\eta_{b r}$ should be negative. The phase of the breaking, $x_{b r}$, may be simply found as

$x_{b r}=\pi-\arcsin \left(\frac{\eta_{b r}}{a}\right)+2 \pi n, \quad n=1,2,3, \ldots$

In the case of equal depths of the layers solutions (38) and (39) are greatly simplified and give

$\eta_{b r}=-\frac{a}{\sqrt{2}}, \quad x_{b r}=\frac{5 \pi}{4} \quad\left(\right.$ when $\left.h_{10} \rightarrow 1 / 2\right)$.

Actually, in this case a second breaking point appears, as has been noted: $\eta_{b r}=2^{-\frac{1}{2}} a$ and $x_{b r}=\pi / 4$; the wave properties in this point are just symmetric reflection and will not be discussed individually.

The dependences defined by (38) and (39) are illustrated in Figs. 4 and 5. Qualitatively the curves from Fig. 4 are very similar to the ones plotted in Fig. 5, which naturally follows from the form of formula (39). The relation (40) gives the horizontal dashed lines in Figs. 4, 5; the other limit $h_{10} \rightarrow 0$ is given by the dotted black lines in the figures. A smallamplitude wave breaks near the rest level, when $x=\pi$, while very intense waves break close to the troughs; this is more significant when the layers have very different depths. In the limiting case when $h_{10} \rightarrow 0$

$\eta_{b r}=-a, x_{b r}=3 \pi / 2\left(\right.$ when $\left.h_{10} \rightarrow 0\right)$,

thus, the wave touches the bottom and breaks at this point. The maximum waves always break at levels $\eta_{b r} \leq-2^{-\frac{1}{2}} a$ on the front slope below the rest level $\left(5 \pi / 4 \leq x_{b r} \leq 3 \pi / 2\right)$.

The breaking time $t_{b r}$ may be found from (34) with the use of (35) computed at the breaking point defined by (39). The dependences of the "breaking distance" $t_{b r} V_{\text {lin }}$ versus normalized wave amplitude $a / h_{10}$ are shown in logarithmic coordinates in Fig. 6. It is interesting to note that even for the 


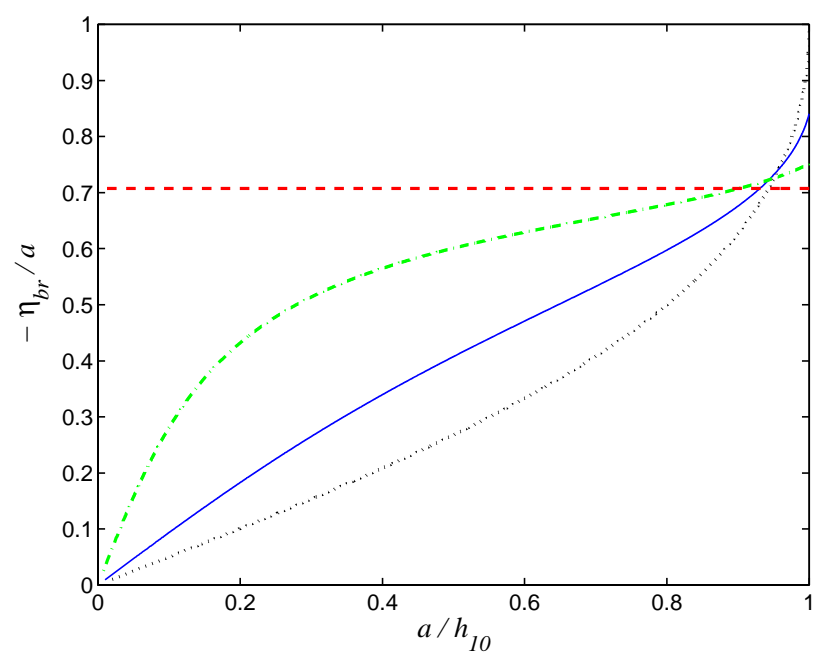

Fig. 4. Relations between the relative wave amplitude $a / h_{10}$ and the position of the breaking point on the sinusoidal wave profile for the cases $h_{10} \rightarrow 0$ (black dots), $h_{10}=0.1$ (solid blue line), $h_{10}=0.3$ (dash-dotted green line) and $h_{10}=0.5$ (dashed red line).

maximum wave amplitudes (when the wave touches the bottom or the water surface) the breaking distance is not zero, because the quantity $\min \left(V_{0}^{\prime}\right)$ remains finite (see Fig. 2). When $h_{10}$ becomes small, the breaking time $t_{b r}$ even grows, because the linear wave speed decays.

The evolution of a wave during its steepening is described by solution (32), thus it may be trivially calculated. The wave profiles at different time moments are shown in Fig. 7 for the cases $h_{1}=0.1(\mathrm{a}, \mathrm{b}), 0.3(\mathrm{c}, \mathrm{d})$ and $0.5(\mathrm{e}, \mathrm{f})$. The amplitudes of the initial sinusoidal waves are chosen equal to the half of the lower layer $a=h_{10} / 2$ (panels (a), (c) and (e)) and to its total thickness $a=h_{10}$ (panels (b), (d) and (f)). The shapes are given for the initial moment of time $t=0, t=t_{b r} / 2$ and $t=t_{b r}$. It is worth mentioning that the breaking point lies close to the point of zero nonlinear velocity for large wave amplitudes, but does not coincide with it. Two breaking points are readily observed for the case $h_{1}=0.5$ in the positive and negative parts of the interfacial displacement.

The process of the wave deformation is directly related to the energy exchange between harmonics due to the nonlinearity. For the better understanding of this process the Fourier analysis of the evolving waves is performed in the next section.

\section{Spectral analysis}

The spectral analysis of a nonlinear wave evolution reveals energy exchange between harmonics which leads to the change of scales of the waves. Defining the spectral function as

$S(k, t)=\frac{1}{2 \pi} \int_{-\infty}^{\infty} \eta(x, t) e^{-i k x} d x$

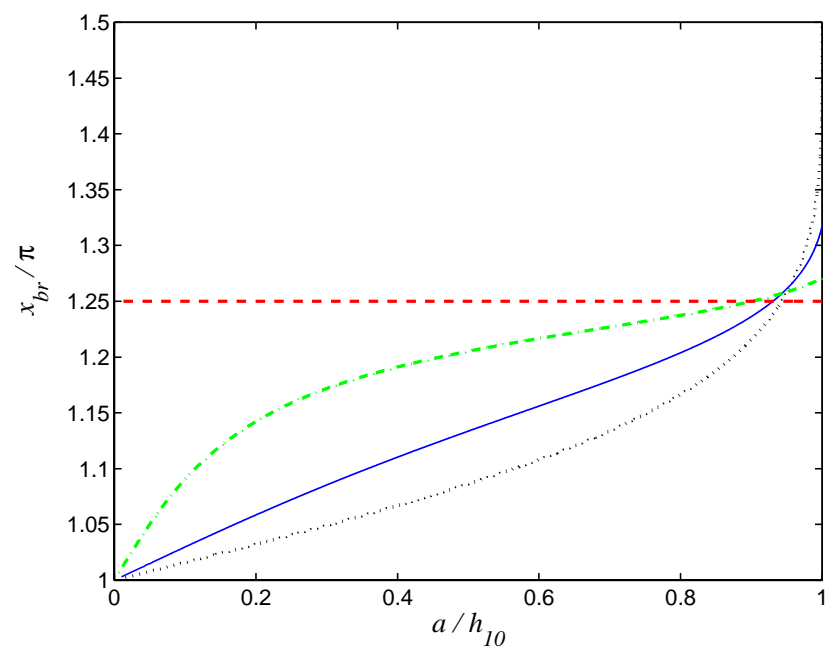

Fig. 5. Relations between the relative wave amplitude $a / h_{10}$ and the phase of the breaking point for cases $h_{10} \rightarrow 0$ (black dots), $h_{10}=0.1$ (solid blue line), $h_{10}=0.3$ (dash-dotted green line) and $h_{10}=0.5$ (dashed red line).

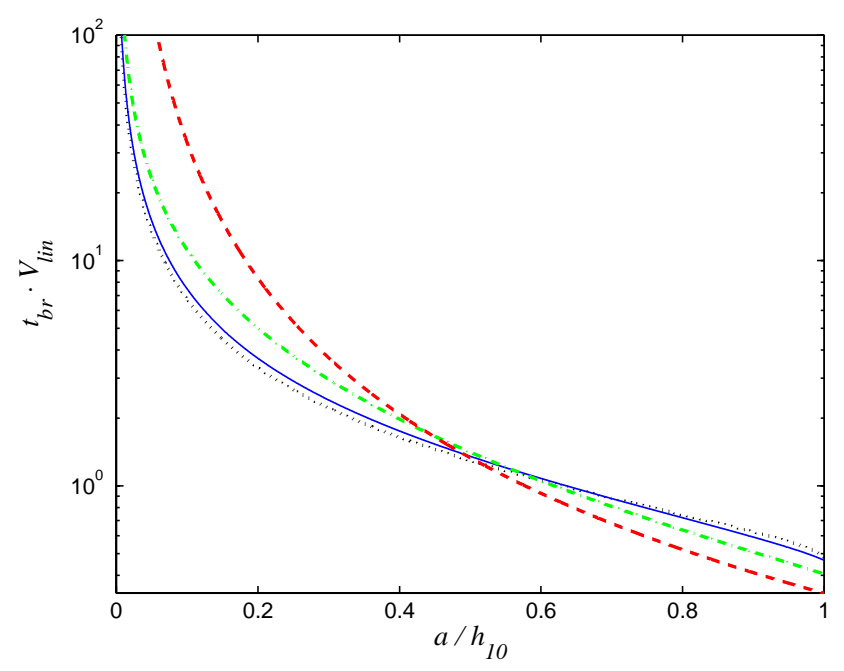

Fig. 6. The breaking distance $t_{b r} V_{\text {lin }}$ versus the relative wave amplitude $a / h_{10}$ for cases $h_{10} \rightarrow 0$ (black dots), $h_{10}=0.1$ (blue solid line), $h_{10}=0.3$ (dash-dotted green line) and $h_{10}=0.5$ (dashed red line).

in the case of the Riemann wave (32) the formula (42) transforms to

$S(k, t)=\frac{-i}{2 \pi k} \int_{-\infty}^{\infty} d z \frac{d \eta_{0}(z)}{d z} \exp \left(-i k\left[z+t V_{+}\left(\eta_{0}(z)\right)\right]\right)$.

The other forms of formula (43) convenient for different purposes are given by Pelinovsky (1976). Considering a real periodic sinusoidal wave $\eta_{0}(x)=a \sin (x)$, the spectrum becomes 

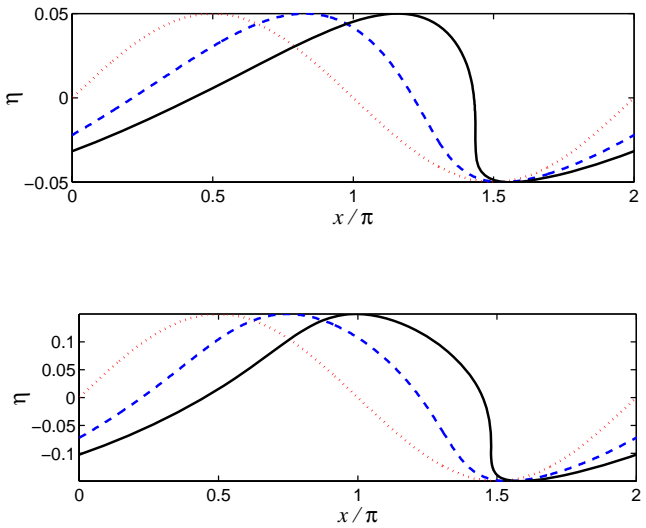

(c)

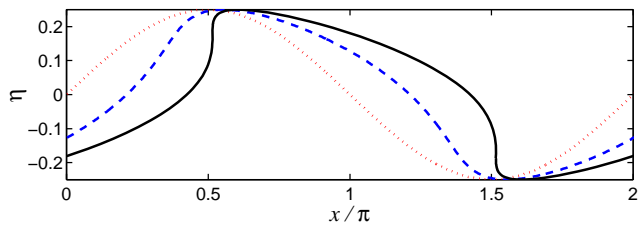

(a)

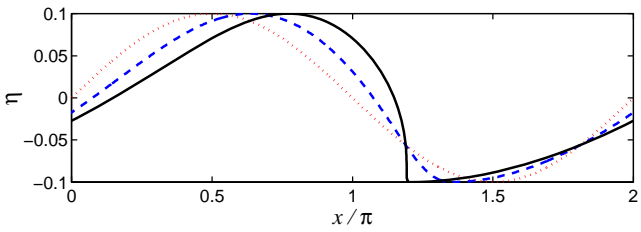

(b)

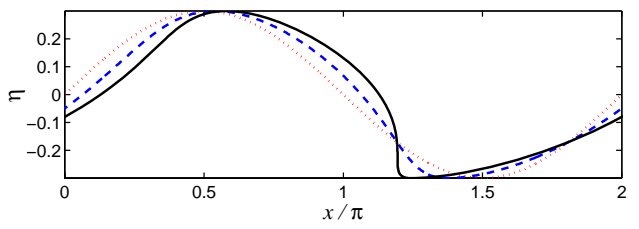

(d)

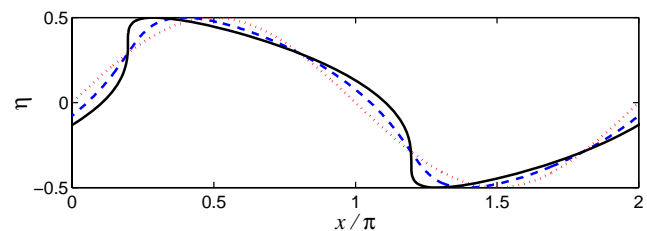

(e)

Fig. 7. The evolution of a sinusoidal wave for different depths of the layers: $h_{10}: 0.1(\mathbf{a}, \mathbf{b}), 0.3$ (c, d) and 0.5 (e, f) and intensity of the waves: $a=h_{10} / 2$ (a, c, e) and $a=h_{10}(\mathrm{~b}, \mathrm{~d}, \mathrm{f})$. Wave shapes are plotted in co-moving reference frames (with speed $V_{\text {lin }}$ ) for the initial moment $t=0$ (dots), $t=t_{b r} / 2$ (dash) and when breaking $t=t_{b r}$ (solid).

complex conjugated: $S(-k)=S(k)$ and discrete $S(k)=S_{k}$. Thus, only positive wavenumbers will be analyzed, as $k=1,2,3, \ldots$ Then the formula for the spectrum is

$S_{k}(t)=-i \frac{a}{\pi k} \int_{0}^{2 \pi} d \xi \cos (\xi) \exp \left(-i k\left[\xi+t V_{+}(a \sin (\xi))\right]\right)$

The expression for the nonlinear velocity (24) has a rather complicated form. For qualitative comprehension it may be useful to represent the nonlinear velocity in powers of the displacement (form (29)). The integral (44) was evaluated by Pelinovsky (1976) in terms of special functions for the power-law nonlinearity $V \sim \eta^{n}$. (It is convenient to study the waves in a reference frame co-moving with the linear velocity $V_{\text {lin }}$, hence we omit the unit in (29).) In particular, in the case $V_{+}=\alpha V_{\text {lin }} \eta$ the spectrum is purely imaginary, and thus the wave field is represented by the series of sinusoids:

$$
\begin{aligned}
& \eta(x, t)=\sum_{k=1}^{\infty} P_{k}(t) \sin (k x), \\
& P_{k}=(-1)^{k+1} \frac{2 a}{k V_{0} t} J_{k}\left(k V_{0} t\right), \\
& V_{0}=\alpha V_{l i n} a,
\end{aligned}
$$

where $J_{k}$ is the Bessel function of the first kind, and the spectral amplitude is defined by $\left|S_{k}^{\text {quadr }}\right|=\left|P_{k}\right|$. The formula (45) is the classical Bessel-Fubini solution known in nonlinear acoustics (Rudenko and Soluyan, 1977). For the case of the cubic nonlinearity, $V_{+}=\alpha_{1} V_{\text {lin }} \eta^{2}$ the spectrum is complex and the solution has the form (see Pelinovsky, 1976):

$$
\begin{aligned}
\eta(x, t)= & \sum_{k=0}^{\infty}\left[Q_{k}(t) \sin \left((2 k+1)\left(x-V_{0} t / 2\right)\right)\right. \\
& \left.+R_{k}(t) \cos \left((2 k+1)\left(x-V_{0} t / 2\right)\right)\right], \\
Q_{k}= & \frac{a}{2 k+1} J_{k}\left(\left(k+\frac{1}{2}\right) V_{0} t\right), \\
R_{k}= & \frac{a}{2 k+1} J_{k+1}\left(\left(k+\frac{1}{2}\right) V_{0} t\right), \\
V_{0}= & \alpha_{1} V_{\text {lin }} a^{2} .
\end{aligned}
$$

The spectral amplitude is then defined by $\left|S_{k}^{\text {cub }}\right|=\sqrt{P_{k}^{2}+Q_{k}^{2}}$. One should pay attention to the fact that only odd harmonics are generated in this case. Cosine terms appear in solution (46), although the initial profile was sinusoidal. This fact is responsible for the shift of the first breaking point from the zero-crossing point that has been discussed above.

The evolution of the wave shape due to nonlinear steepening is illustrated in Fig. 7 for different rest levels of the 


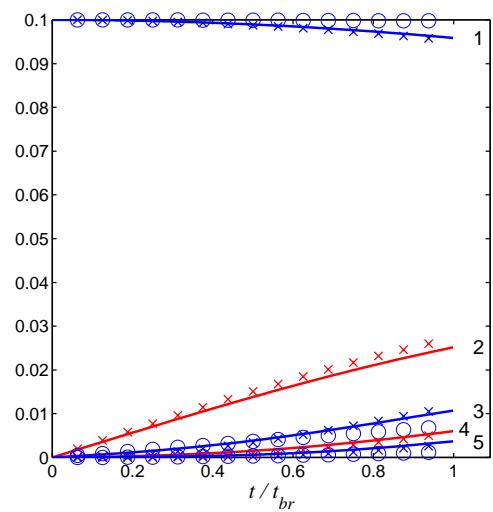

(a)

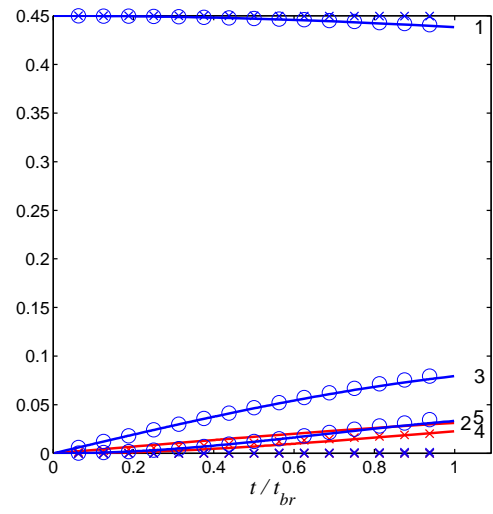

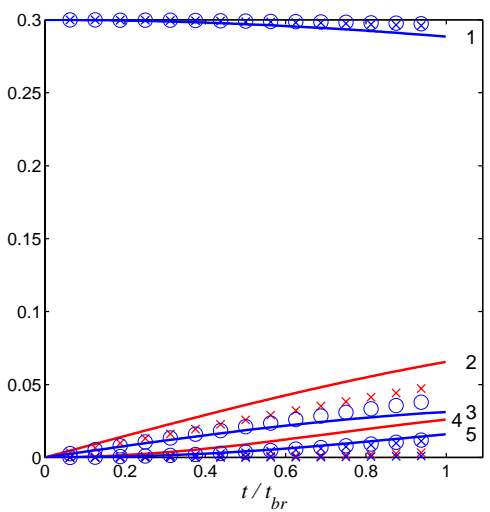

(b)

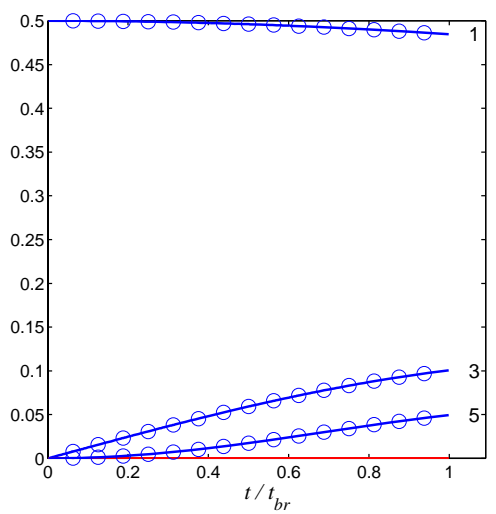

(d)

Fig. 8. The evolution of the amplitudes of the first five Fourier modes due to the nonlinear wave evolution (as shown in Fig. 7) for different ratios of the layer depths: $h_{10}=0.1$ (a), $h_{10}=0.3$ (b), $h_{10}=0.45$ (c) and $h_{10}=0.5$ (d). The initial sinusoidal wave has maximum amplitude $a=h_{10}$. Solid lines show the numerical solution $\left|S_{k}(t)\right|$ (ordinal numbers of harmonics are given nearby). The markers show analytical

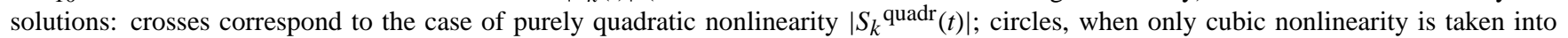
account $\left|S_{k}{ }^{\operatorname{cub}}(t)\right|$.

interface and different intensities of the waves. The spectral evolutions of the maximum $\left(a=h_{10}\right)$ waves for that cases (plus one extra case $h_{10}=0.45$ ) are shown in Fig. 8, where the first five spectral coefficients $\left|S_{k}(t)\right|$ are plotted by solid lines (numerical integration of (44)). For the considered cases when the interfacial boundary is placed in the lower half of the water column $\left(h_{10} \leq h_{20}\right)$ the coefficient of quadratic nonlinearity $\alpha$ is positive and the cubic nonlinear coefficient $\alpha_{1}$ is negative (see (30)).

The quadratic nonlinearity plays the major role when the layers have different depths (one may compare terms in (29) taking $\eta=\mathrm{h}_{10}$ ). Therefore Fig. 7 (a-d) demonstrate asymmetric deformation of the wave according to the nonlinear corrections to the velocity (Figs. 2a, b) with the formation of one breaking point (on the front face between the rest level and the wave trough).

In the case of almost equal thicknesses of the layers $\left(h_{10} \rightarrow 1 / 2\right)$, the quadratic nonlinear term degenerates, and the cubic nonlinear term becomes the leading term in the asymptotic series (29). It symmetrically decreases the wave ve- locity for both positive and negative disturbances (Fig. 2c). The limiting case $h_{10}=0.5$ corresponds to a simpler (squared) dependence of the nonlinear velocity (31). There are two minima of function $d V_{0} / d x$ (Figs. $3 \mathrm{e}, \mathrm{f}$ ) due to the symmetry with respect to the horizontal line, and thus there are two first breaking points: above and below the rest level (Figs. 7e, f).

The solutions of (45) and (46) are plotted in Fig. 8 with red crosses and blue circles respectively. Purely cubic nonlinearity excites only odd harmonics (Fig. 8d), while other cases generate all Fourier modes. It is understood from these plots that when quadratic nonlinearity is dominant (the interface is not close to the middle of the water depth), the harmonics are ordered by the energy transfer (Figs. 8a, b). When the cubic nonlinear term becomes more important, this order is destroyed: in Fig. 8c the most energetic (after the fundamental) mode is number 3 , then number 5 follows, and number 2 goes after this. Odd harmonics become more energetic, while the total energy transferred from the first harmonic is smaller; even modes are depressed and vanish totally in the case when the layers have equal depths (Fig. 8d). It follows 
from Fig. 8 that the estimates (45) and (46) give reasonable results, at least for several low harmonics, when one of the nonlinear terms (quadratic or cubic) prevails.

\section{Conclusions}

In this paper the nonlinear interfacial gravity wave transformation is studied in the Boussinesq assumption, and when the dispersive effects are neglected. Explicit formulation of the evolution equation in terms of the Riemann invariants allows us to obtain analytical results characterizing strongly nonlinear wave steepening, including the spectral evolution. This dynamics is considered to be the first stage resulting in wave breaking. Effects showing the action of highly nonlinear corrections of the model are highlighted. It is shown, in particular, that the breaking points on the wave profile may shift from the zero-crossing level; wave steepening occurs differently in the case when the density jump is placed near the middle of the water column: then the wave deformation is almost symmetrical and two breaking wave phases exist.

Acknowledgements. This work was supported by grants INTAS 0351-4286 and 06-1000013-9236 (E.P., T.T., A.K., O.P., A.S.); RFBR 05-05-64333, 06-05-64087 (O.P., A.K.), and 06-05-64232 (T.T., A.S.); grant MK-798.2007.5 and Russian Science Support Foundation (A.S.).

Authors are grateful to the Editor for improving the English language of the manuscript.

Edited by: R. Grimshaw

Reviewed by: Y. Stepanyants and another referee

\section{References}

Afanasyev, Ya. D. and Pelter, W. R.: On breaking internal waves over the sill in Knight Inlet, Proc. Royal Society London, A457, 2799-2825, 2001.

Armi, L. and Farmer, D.: Stratified flow over topography bifurcation fronts and transition to the uncontrolled state, Proc. Royal Society London, A457, 2827-2830, 2001.

Baines, P. G.: Topographic effects in stratified flows, Cambridge University Press, 1995.

Chant, R. and Wilson, R.: Internal hydraulics and mixing in highly stratified estuary, J. Geophys. Res., 105(C6), 14215-14222, 2000.

Craik, A. D.: Wave interactions and fluid flows, Cambridge University Press, 1985.
Fringer, O. B. and Street, R. L.: The dynamics of breaking progressive interfacial waves, J. Fluid Mech., 494, 319-353, 2003.

Grimshaw, R. (Ed): Environmental stratified flows, Kluwer Acad. Publ., 2002.

Helfrich, K. R. and Melville, W. K.: Long nonlinear internal waves, Annu. Rev. Fluid Mech., 38, 395-425, 2006.

Henyey, F. S. and Hoering, A.: Energetic of borelike internal waves, J. Geophys. Res., 102(C2), 3323-3330, 1997.

Holland, D., Rosales, R., Stefanica, D., and Tabak, E.: Internal hydraulic jumps and mixing in two-layer flows, J. Fluid Mech., 470, 63-83, 2002.

Horn, D. A., Imberger, J., and Ivey, G. N.: The degeneration of large-scale interfacial gravity waves in lakes, J. Fluid Mech., 434, 181-207, 2001.

Kakutani, T. and Yamasaki, N.: Solitary waves on a two-layer fluid, J. Phys. Soc. Japan, 45, 2, 674-679, 1978.

Klemp, J. B., Rottuno, R., and Skamarock, W.C.: On the propagation of internal bores, J. Fluid Mech., 331, 81-106, 1997.

Lyapidevsky, V. Yu. and Teshukov, V. M.: Mathematical models of long wave propagation in inhomogeneous fluid, Nauka, Novosibirsk, (in Russian) 2000.

Milewski, P. A., Tabak, E. G., Turner, C. V., Rosales, R. R. and Menzaque, F.: Nonlinear stability of two-layer flows, Comm. Math. Sci., 2, 427-442, 2004.

Miropol'sky, Yu. Z.: Dynamics of internal gravity waves in the ocean, Kluwer, 2001.

Munk, W. and Wunsh, C.: The Moon and mixing abyssal recipes II, Deep-Sea Res. I, 45, 1977-2010, 1998.

Ostrovsky, L. A. and Grue, J.: Evolution equations for strongly nonlinear internal waves, 15, 10, 2934-2948, 2003.

Pelinovsky, E. N.: Spectral analysis of simple waves, Radiophysics and Quantum Electronics, 19, 3, 262-270, 1976.

Polukhina, O. E. and Slunyaev, A. V.: An improved evolution model on the basis of the Gardner equation for internal waves in a stratified fluid, Izvestiya Russian Academy Engineering Science, 18, 82-90, (in Russian) 2006.

Rudenko, O. V. and Soluyan, S. I.: Theoretical foundations of nonlinear acoustics, New York, Consultants Bureau, 1977.

Sakai, T. and Redekopp, L. G.: Models for strongly-nonlinear evolution of long internal waves in a two-layer stratification, Nonlin. Processes Geophys., 14, 31-47, 2007, http://www.nonlin-processes-geophys.net/14/31/2007/.

Slunyaev, A., Pelinovsky, E., Poloukhina, O. and Gavrilyuk, S.: The Gardner equation as the model for long internal waves, Proc. Int. Symp. "Topical Problems of Nonlinear Wave Physics" (Nizhny Novgorod), 368-369, 2003.

Witham, G. B.: Linear and nonlinear waves, John Wiley \& Sons, 1974. 